\title{
A Scrutinized Analysis Method of the Human Error Potential due to the Introduction of New Digital Devices to Nuclear Power Plants
}

\author{
Lee and Yong Hee \\ I\&C and Human Factors Division, \\ Korea Atomic Energy Research Institute (KAERI), \\ 1045 Daeduk-Daero, Yoseong-Gu, Daejeon, 305-353, Korea \\ yhlee@kaeri.re.kr
}

\begin{abstract}
This paper describes a new method proposed for human error analysis (HEA) in case of the introduction of new digital devices to nuclear power plants (NPPs). Preventing human errors has been a main issue especially for the safety of NPPs. The hi-touch human interface must be proved to be safe before being introduced to NPPs. Although many good HEA methods are available, a scrutinized one is inevitable against to the new types of errors, for utilizing new digital devices and their human interfaces in NPPs. All possible interactions between the device and the user through the designed interfaces are investigated by the term of Interaction Segment (IS), and Error Segment (ES) is assessed by human factors criteria and their consequences to predict the human error potential (HEP). Results such as a list of ISs and ESs and their combinatorial sequences are provided to the human interface designers for qualifying the digital devices to NPPs.
\end{abstract}

Keywords: human error, human interface, digital device, nuclear power plant.

\section{An Approach to Human Errors in Digital Human Interface}

Human error has been notorious as a universal enemy which has potential to break down all barriers and protective features for system safety. Many researchers have investigated the task performance of human and its influence to overall level of functional reliability. They have performed the studies to predict and prevent the human error through probability and usability; however, inadequate decisions and/or unwanted behaviors are inevitable to human itself. It has been complicated to know the details mechanism of human errors previously as the high technology digital instruments have been developed and introduced. Thus, it was needed to predict the detailed error potential, and to prevent previously in design of, especially NPPs. ES and IS were defined according to exterior physical units and operation options of the digital devices. It could be possible to review all interactions through ES and IS for using digital devices.

This paper describes a new method proposed for human error analysis (HEA) in case of the introduction of new digital devices to nuclear power plants (NPPs). 
Recently, the human interface has been drastically revolved by virtue of the hi-touch technology as well as the digital and computer technology. It is still strongly doubtful to find such an up-to-date interface in NPPs in near future. Every new design and new technologies must be proved to be safe before introducing to NPPs, and free from defects including all kinds of human errors.

The human error potential within the digital devices should be thoroughly investigated from the early phase of design to the integration and operational phase. Although many good HEA methods are available, a scrutinized one is inevitable against to the new types of errors, for utilizing new digital devices and their human interfaces in NPPs.

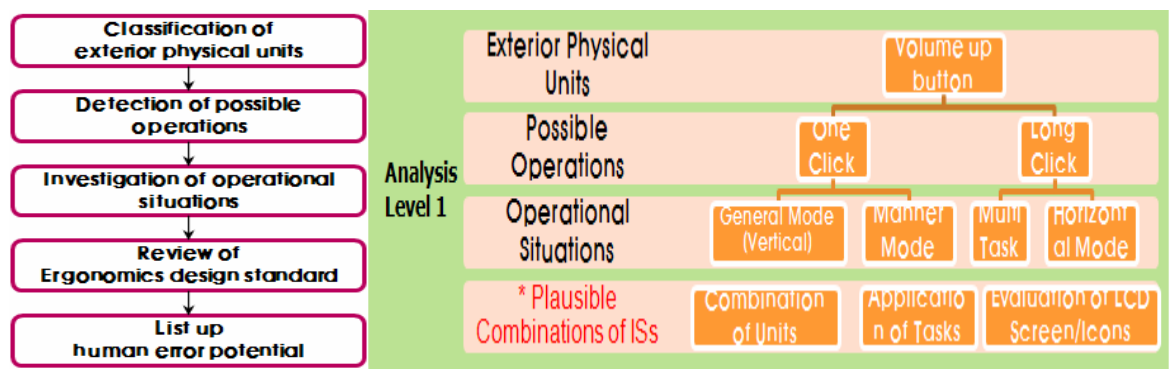

Fig. 1. Steps for HEA proposed

\section{Interaction Segments (IS) and Error Segments (ES)}

A digital device has various design factors of interface itself that might give very different impact to the user by not only the shape and color but also the operation methods, control states, and so on. Lee, et al. (2003) defined Error Segment as "the basic unit has the potential causing negative affect for use"[1]. In this study to find out the human error potential (HEP), two new terms such as Interaction Segment (IS) and Error Segment (ES) are introduced. All possible interactions between the device and the user through the designed interfaces are described in term of Interaction Segments. And Error Segments are assessed among the various combinations of ISs. They are enumerated into the combinatorial structures of all applicable tasks, nevertheless their frequencies might be extremely low.

The human error potentials of each $I S / E S$ are respectively examined by human factors engineering criteria, and the plausible sequences of various combination of all ISs are assessed by their evolutionary paths to the ultimate consequences. Results such as a list of Error Segments (ESs) and their combinatorial sequences are provided to the human interface designers for qualifying the digital devices to NPPs.

\section{Example Application and the Results}

Three kinds of smart phones were analyzed and they are named type A, B, C. It has been using with the limited function in NPPs currently [2]. ES and IS were defined from instrument (device character, $\mathrm{H} / \mathrm{W}$ and $\mathrm{S} / \mathrm{W}$ design problem, and component 
breakdown), user (operation method, knowledge level, and user character), operation situation and so on. ES was classified by exterior physical units of the smart phone and IS was listed from user and operation situation. (LCD buttons are excluded just they are reviewed as an integrated icon in the applications.) They will be considered to a further research. Followings are results of example application of the proposed method to three different types of smart-phones for design review before NPP field applications. Table 1 shows a part of IS lists coded at capital alphabet. Figure 1 shows a comparative result about the total numbers of ISs. It is quite clear that type $\mathrm{C}$ smart phone has less exterior physical units and less ISs than the others.
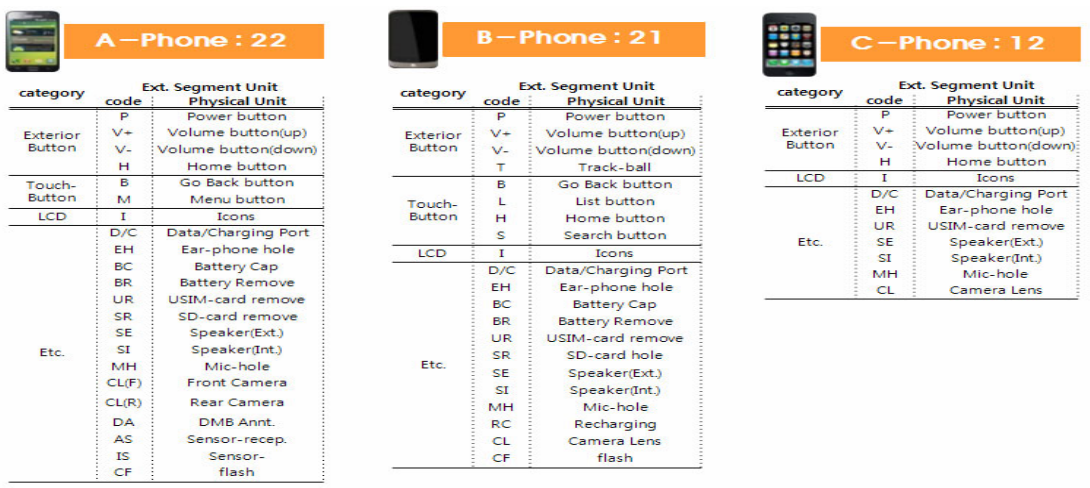

Fig. 2. The example of IS and ES for A and B type smart phone

Table 1. List of IS and ES (a part for smart phone)

\begin{tabular}{|c|c|c|c|c|c|c|}
\hline \multirow{2}{*}{$\begin{array}{l}\text { IS } \\
\text { Code. }\end{array}$} & \multirow{2}{*}{$\begin{array}{l}\text { Operation } \\
\text { method }\end{array}$} & \multicolumn{5}{|c|}{ Test for ES by Operation situation } \\
\hline & & No option(Vertical) & Manner mode & Horizontal mode & Multi task & Lock \\
\hline \multirow{2}{*}{$P$} & One Click & \multicolumn{5}{|c|}{ Screen on/off } \\
\hline & Long Click & \multicolumn{5}{|c|}{ Pup-up window for option } \\
\hline \multirow{2}{*}{$\mathrm{V}+$} & One Click & Vol. up by stages & $\begin{array}{l}\text { Manner mode cancel } \\
\text { and vol. up by stages }\end{array}$ & \multirow{2}{*}{$\begin{array}{l}\text { Vol. up by stages } \\
\text { (space } \\
\text { compatibility) } \\
\text { Oper. intended: } \\
\text { down }\end{array}$} & & None \\
\hline & Long Click & Vol. up rapidly & $\begin{array}{l}\text { Manner mode cancel } \\
\text { and vol. up rapidly }\end{array}$ & & & None \\
\hline \multirow[t]{2}{*}{ V - } & One Click & $\begin{array}{l}\text { Vol. down by } \\
\text { stages } \rightarrow \text { manner } \\
\text { mode }\end{array}$ & Vibration & \multirow{2}{*}{$\begin{array}{c}\text { Vol. down by stages } \\
\text { (space } \\
\text { compatibility) } \\
\text { Oper. intended: up }\end{array}$} & & None \\
\hline & Long Click & $\begin{array}{l}\text { Vol. down rapidly } \\
\rightarrow \text { manner mode }\end{array}$ & Vibration & & & None \\
\hline \multirow{2}{*}{$\mathrm{T}$} & Rotation & Move & Move & Move & Move & None \\
\hline & Click & Selection & Selection & Selection & Selection & None \\
\hline
\end{tabular}

For an example, in case of HEP at ' $\mathrm{V}+$ ' in ES and 'Horizontal mode' in IS, operators have different intention about Volume up button $(\mathrm{V}+)$ between no option and horizontal mode in IS. However they showed the same response. When $\mathrm{V}+$ is in horizontal mode, it has performed to turn down the volume (V-). It caused confusion to operators by violating the space compatibility. If $\mathrm{V}+$ is in horizontal mode, volume would be decreased by stage or rapidly. Because $\mathrm{V}+$ is in the left of the device at horizontal mode, usually operators are likely to push the left button for decreasing the volume. The application includes a review by design criteria from the human factors 
engineering/ergonomics design standards such as functional availability, suitability of design, consistency, compatibility, effectiveness for use, and so on.(see Figure 2).
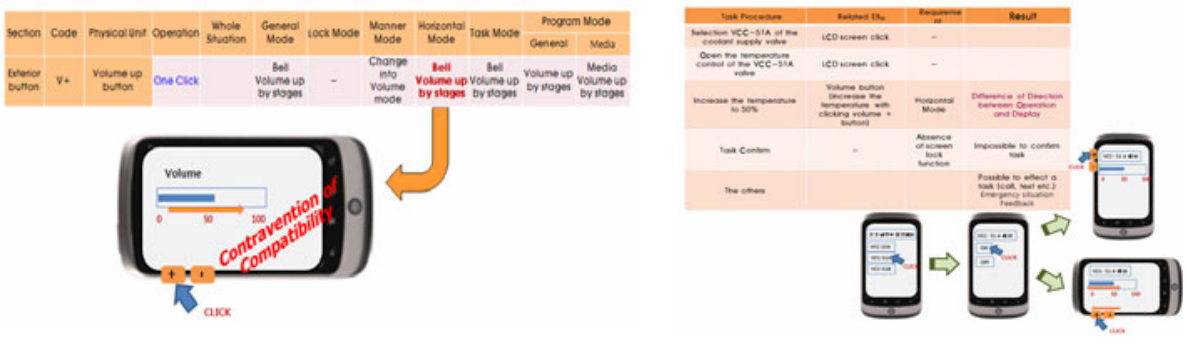

Fig. 3. Review on the interface by the human factors criteria
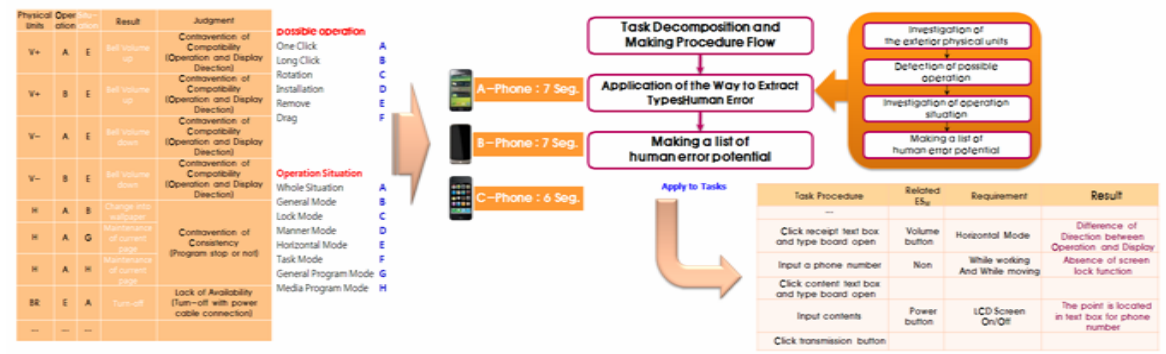

Fig. 4. Steps and results according to the applications to smart-phones

\section{Conclusions and Discussions}

A new HEA method summarized in figure 3 can facilitate to specify HEP of a digital device based on the Error Segment (ES) and Interaction Segment (IS). All possible interactions can be described in term of IS/ES, and HEP can be thoroughly investigated from the early phase of design by providing a list of IS/ESs to the human interface designers for qualifying the digital devices to NPPs. The results are to be verified through the experimental observation [3].

\section{References}

1. Lee, Y.H., Jang, T.I., Lim, H.K.: A modification of human error analysis technique for designing man-machine interface in nuclear power plants. Journal of the Ergonomics Society of Korea 22(1), 31-42 (2003)

2. Yun, J.H., Oh, Y.J., Lee, Y.H.: A development of the Extremely-Low Frequency Human Errors of Digital Devices. In: Proceeding of Conf. Ergonomics Society of Korea (2010)

3. Oh, Y.J., Lee, Y.H., Yun, J.H.: A Study on the Operator's Erroneous Responses to the New Human Interface of a Digital Device to be introduced to Nuclear Power Plants. In: Posters, Part I, HCII 2011. CCIS, vol. 173, pp. 342-345. Springer, Heidelberg (2011) 